\title{
Experimental Results in Synchronous-Clock One-Way-Travel-Time Acoustic Navigation for Autonomous Underwater Vehicles
}

\author{
Ryan M. Eustice*, Louis L. Whitcomb ${ }^{\dagger}$, Hanumant $\operatorname{Singh}^{\ddagger}$, and Matthew Grund ${ }^{\ddagger}$ \\ *Department of Naval Architecture \& Marine Engineering \\ University of Michigan, Ann Arbor, MI 48109 \\ email: eustice@umich.edu \\ ${ }^{\dagger}$ Department of Mechanical Engineering \\ Johns Hopkins University, Baltimore, MD 21218 \\ email: llw@jhu.edu \\ ‡Department of Applied Ocean Physics \& Engineering \\ Woods Hole Oceanographic Institution, Woods Hole, MA 02543 \\ email: \{hanu,mgrund\}@whoi.edu
}

\begin{abstract}
This paper reports recent experimental results in the development and deployment of a synchronous-clock acoustic navigation system suitable for the simultaneous navigation of multiple underwater vehicles. The goal of this work is to enable the task of navigating multiple autonomous underwater vehicles (AUVs) over length scales of $\mathcal{O}(100 \mathrm{~km})$, while maintaining error tolerances commensurate with conventional longbaseline transponder-based navigation systems (i.e., $\mathcal{O}(1 \mathrm{~m})$ ), but without the requisite need for deploying, calibrating, and recovering seafloor anchored acoustic transponders. Our navigation system is comprised of an acoustic modem-based communication/navigation system that allows for onboard navigational data to be broadcast as a data packet by a source node, and for all passively receiving nodes to be able to decode the data packet to obtain a one-way travel time pseudo-range measurement and ephemeris data. We present results for two different field experiments using a two-node configuration consisting of a global positioning system (GPS) equipped surface ship acting as a global navigation aid to a Doppler-aided AUV. In each experiment, vehicle position was independently corroborated by other standard navigation means. Initial results for a maximumlikelihood sensor fusion framework are reported.
\end{abstract}

\section{INTRODUCTION}

Few techniques presently exist for reliable threedimensional position sensing for underwater vehicles. Depth, altitude, heading, and roll/pitch attitude can all be instrumented with high bandwidth internal sensors. XY position, in contrast, remains difficult to instrument and is normally measured acoustically in oceanographic and commercial applications.

Conventional long-baseline (LBL) acoustic navigation systems require multiple fixed transponders - i.e., fixed or moored on the seafloor [1], [2], on the hull of a surface ship [3], or on sea-ice [4]. With a maximum acoustic range of 5-10 km, fixed LBL networks can cover only limited mission areas. Moreover, existing LBL navigation systems are designed to navigate one vehicle per interrogation-response acoustic cycle using a time division multiple access (TDMA) scheme. This is acceptable for single vehicle deployments, but less desirable for multi-vehicle deployments because the interrogation-response navigation update period increases linearly with the number of vehicles (thereby proportionally decreasing each vehicle's overall navigation update rate). In practice, this limits multi-vehicle LBL navigation to networks of a few vehicles. The existing prevalence of LBL systems within the oceanographic community is due to a lacuna of alternative means for obtaining bounded-error subsea XY position.

While the advent of the global positioning system (GPS) provides bounded-error terrestrial navigation for both surface and air vehicles, seawater is opaque to the radio-frequencies upon which GPS relies and, thus, GPS cannot be used by submerged underwater vehicles. Though ultra-short-baseline (USBL) acoustic navigation systems are preferred for shortrange navigation, they are of limited usefulness for longrange navigation [5], [6] and, furthermore, also suffer from the same TDMA update problem as LBL.

Meanwhile, the high cost and power consumption of inertial navigation systems (INSs) has, until now, precluded their widespread use in non-military undersea vehicles. Compact, low-cost, low-power INS systems have recently become commercially available, offering an alternative method for instrumenting absolute XYZ displacement [7], [8]. Modern INS position error is on the order of $1 \%$ of path-length, hence, INS alone is inadequate to support the needs of long-range bounded-error navigation. For example, the path length of a vehicle traveling 3 knots for 48 hours is $144 \mathrm{~nm}$ $(266 \mathrm{~km})$, resulting in an INS position error of $1.4 \mathrm{~nm}$ $(2.6 \mathrm{~km})$, which is unacceptably large. For a survey of current underwater navigation technologies, the reader is referred to [9].

In this paper we pursue the development of a synchronousclock acoustic modem-based navigation system capable of supporting multi-vehicle bounded-error navigation over large 
length scales (e.g., $\mathcal{O}(100) \mathrm{km})$. Our navigation framework employs WHOI Micro-Modems [10], [11], an underwater acoustic modem developed by Woods Hole Oceanographic Institution (WHOI), in conjunction with commercial off-theshelf (COTS) low-power stable clocks to yield a navigation system capable of inter-vehicle communication and oneway travel time (OWTT) ranging. Previous work using modems and synchronous-clock navigation has been reported in [12] for autonomous surface-craft; in that work each vehicle was equipped with a GPS receiver to provide a common timebase for synchronous ranging. Synchronous-pinger OWTT navigation was reported in [13] where integrated range-rate positioning was used for autonomous underwater vehicle (AUV) docking using an early predecessor of the modern WHOI Micro-Modem. Non-modem synchronousclock OWTT ranging has been reported in [2] for "in-hull navigation" of the manned deep-submergence vehicle Alvin. Other loosely related work involving single transponder twoway travel-time (TWTT) navigation has been reported in [14]-[20].

The rest of the paper is organized as follows. Section II describes our synchronous-clock implementation and methodology. Section III presents a stochastic sensor fusion framework for combining OWTT pseudo-range measurements with strap-down onboard vehicle Doppler odometry for bounded-error navigation. Section IV reports field results for two different sets of data collected in-situ by a bottomlock Doppler-aided AUV accompanied with OWTT pseudorange measurements acquired from a GPS-equipped surface ship. Finally, section V offers some concluding remarks.

\section{Synchronous-Clock Acoustic NAVigation}

Most acoustic navigation systems are based upon measuring two-way time-of-flight (TOF) ranges whereby the navigation cycle consists of a vehicle-initiated interrogation pulse followed by a reply from all passively listening transponders [2], [3]. In these systems, each node (i.e., vehicle) in the acoustic network must interrogate the network in order to obtain a TWTT measurement between it and all replying nodes. The advantage of such a system is that no absolute timebase is required for TOF measurement, however, the disadvantage is that in a $N$ vehicle environment the overall update rate for each vehicle decreases as $\frac{1}{N}$. Moreover, only the interrogating node has observability of the TWTT measurement used to compute the navigation fix.

In contrast, accurate OWTT ranging can be determined by knowing precisely the transmit and receive times of an underwater acoustic communications packet. The result is a direct one-way TOF measurement from source to receiver. The advantage over TWTT ranging is that OWTT ranging readily scales to a multi-vehicle environment within a master/slave architecture (where inter-vehicle communication is not required). This is because when a source (master) node interrogates the network, all receiving nodes (slaves) can passively measure the one-way TOF to the source node. Hence, in a $N$ vehicle environment, the overall update rate for each (slave) vehicle remains constant. The disadvantage, however, is increased complexity in hardware design since all nodes must carry their own synchronized stable clock.

\section{A. Methodology}

Our methodology is to use OWTT ranging capabilities in the context of a surface-ship acting as a moving transponder. For this purpose, we are employing the synchronous-transmission capabilities of the WHOI Micromodem [10], [11], [21]. The synchronous-transmission feature of the WHOI Micro-modem allows it to directly and accurately measure time-of-arrival (TOA) to within $125 \mu \mathrm{s}$ (i.e., $18.75 \mathrm{~cm}$ precision at a sound speed of $1500 \mathrm{~m} / \mathrm{s}$ ) between a source and receiver using a user supplied external pulse per second (PPS) reference. This common timebase allows for a synchronous modem communication/navigation system whereby navigation data packets can encode time of origin information as well as local ephemeris data (e.g., XYZ positional data and error metric). Navigation packets can be acoustically broadcast to the vehicle network, allowing all receiving nodes to passively measure the elapsed TOF to the source node. The OWTT pseudo-range knowledge, when used in conjunction with the decoded ephemeris data and other onboard vehicle navigation data, provides a mechanism for bounded-error self-localization.

In our method, a ship maneuvers with an AUV fleet, tending to vehicle launch/recovery support, while also acting as a global navigation aid by broadcasting GPS-derived ship transducer position to the vehicle network. All vehicles which are within listening range of the ship and which passively receive the GPS ephemeris can then use this knowledge to compute a running position fix and correct accumulated dead-reckoning error. For this purpose, we anticipate that vehicles will be instrumented with a standard suite of oceanographic navigation sensors including pressure depth, attitude, Doppler velocity log (DVL), and possibly an INS.

\section{B. Implementation}

In our system, each submerged node is equipped with a COTS low-power $(10 \mathrm{~mW})$ temperature compensated crystal oscillator (TXCO) manufactured by SeaScan Inc. This freerunning TXCO is capable of providing a $0.02 \mathrm{ppm}$ (typical) (i.e., a drift-rate of about $1 \mathrm{~ms}$ per $14 \mathrm{~h}$ ) reference pulse at the rate of $1 \mathrm{PPS}$. This translates into a maximum per-dive drift-induced range-error of $1.5 \mathrm{~m}$, which is commensurate with standard $12 \mathrm{kHz}$ LBL. The TXCO is naturally freerunning, therefore, we designed a micro-controller-based daughter card, called a PPSBOARD, to provide higher-level clock functionality.

The PPSBOARD mates to the TXCO and controls synchronization of the TXCO's PPS to an absolute reference clock as well as measures the TXCO's PPS offset with respect to the reference. This capability allows us to synchronize the TXCO to a common timebase at the beginning of a mission while on the surface, such as GPS-derived Coordinate Universal Time (UTC)), and then measure the pre and post dive drift of the TXCO, which is useful for measuring clock drift. Additionally, the PPSBOARD generates a NMEA 


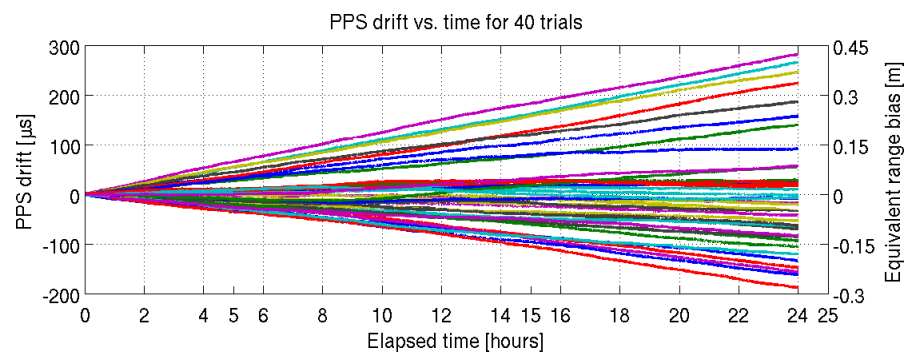

(a)

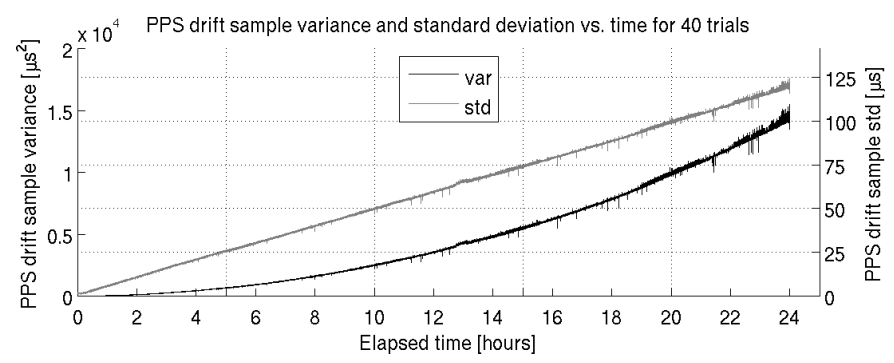

(c)

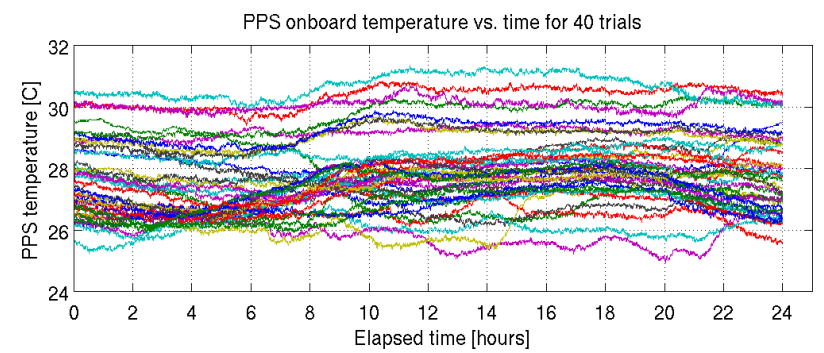

(b)

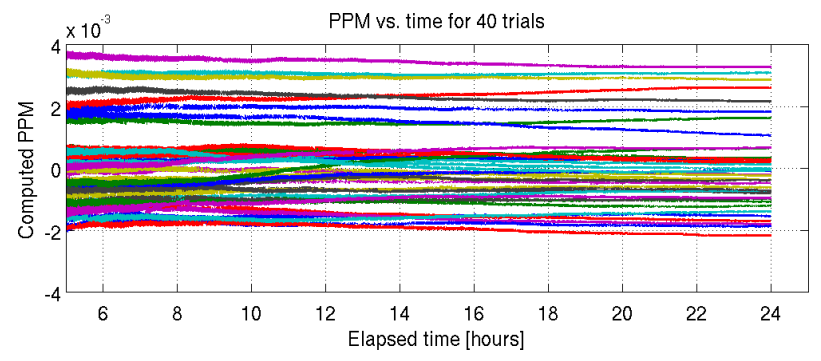

(d)

Fig. 1. PPS drift characterization; benchmark results for 40 trials. Each trial was conducted over a $24 \mathrm{hr}$ period at ambient room temperature. The time axis shows the elapsed time in hours since synchronization of the PPSBOARD with respect to a reference drift-free PPS. (a) Measured PPS drift for all trials; each sample path is color coded by trial number. The $y$-axis on the left reports the measured offset in microseconds while the axis on the right reports it in range equivalent meters (assuming a sound speed of $1500 \mathrm{~m} / \mathrm{s}$ ). (b) Measured onboard TXCO temperature; fluctuation is about normal ambient room temperature. (c) PPS drift sample variance and standard deviation across all trials. (d) Measured PPS drift for all trials, reported in units of ppm.

string and waveform that can be used with the network time protocol (NTP) algorithm [22] to keep real-time clock (RTC) drift onboard the vehicle host PC to within sub-milliseconds of absolute time. This level of accuracy is sufficient for the host PC to be able to assign which second corresponds to which TXCO PPS pulse when communicating with the Micro-Modem. This time reference is then embedded as the time of origin in the acoustic navigation packet.

The surface ship uses a COTS GPS-based network timeserver for a stable clock source. The unit, manufactured by Meinberg Inc., uses a high-quality oven compensated crystal oscillator (OXCO) with a GPS-synchronous accuracy of $1 \mathrm{E}-6 \mathrm{ppm}$ and a free-running accuracy of $5 \mathrm{E}-4 \mathrm{ppm}$. Hence, the ship-based timeserver can be considered essentially driftfree so that only the vehicle's TXCO drift must be accounted for when computing pseudo-range measurements from TOF data.

\section{TXCO PPS Drift Characterization}

To characterize the drift performance of the TXCO used on sub-sea nodes, we performed a series of 40 experiments whereby we synchronized and recorded the free-run drift of the PPSBOARD over a 24-hour period in an ambient room temperature environment. For comparison, we used the Meinberg's PPS signal as ground-truth. Fig. 1 summarizes the outcome of the 40 trials and displays measured drift, sample statistics, and recorded ambient temperature for each run. In particular, note in Fig. 1(a) the time-varying drift phenomenon of the TXCO PPS. Qualitatively, the timevarying nature of the sample paths appears similar to that of a random-walk process. Quantitatively, however, the associated sample variance does not exhibit a linear growth with time (Fig. 1(c)), as would be predicted by theory if it were indeed an i.i.d. process. Instead it appears to exhibit quadratic growth with time (linear standard deviation). At this point in time, further analysis is required to properly model clock drift.

The maximum drift error across all trials in Fig. 1(a) is under $300 \mu \mathrm{s}$, which translates into a drift-induced range bias of less than $0.45 \mathrm{~m}$ over a $24 \mathrm{~h}$ period. While the experiments reported here were for an ambient temperature environment, we have also conducted some preliminary studies in which we immersed the TXCO into a controlled temperature-bath - exposing it a step-response temperature change. Initial results confirm that the magnitude of error drift remains within the manufacturer reported error tolerance of $0.02 \mathrm{ppm}$. This is relevant because PPS synchronization will typically occur at the surface prior to launch, meaning that the vehicle electronics and enclosed TXCO will go through a temperature gradient between ambient air at the surface to seafloor water temperature at depth. So while we should expect the drift performance of the TXCO to degrade with respect to the controlled laboratory results reported here, it still suggests that for relatively short-duration missions (i.e., under several hours in length) drift-induced range-bias will be a negligent error source in overall underwater vehicle navigation.

\section{MAXimum Likelihood Fusion Framework}

In this section, we describe a maximum-likelihood sensor fusion framework for bounded-error $\mathrm{XY}$ vehicle navigation that combines: (i) vehicle-derived inter-ping Doppler 
odometry, (ii) ship GPS-derived position, and (iii) OWTT pseudo-range measurements between ship and vehicle. The framework is suitable for offline batch post-processing for the purposes of optimal re-navigation, and can also be extended to online in-situ vehicle use by selecting an appropriate sliding time window of most recent data.

\section{A. Assumptions}

In the forthcoming formulation we make the following assumptions:

1) First, that dives are relatively short in duration (i.e., on the order of several hours) such that PPS drift-induced range-bias remains negligible over the course of the dive and, therefore, can be neglected.

2) Second, that the sound speed profile is locally homogeneous within the prescribed bounding box of vehicle operations. This implies that TOF measurements can be converted to pseudo-ranges via a linear scaling by sound velocity.

3) Third, that the estimation problem can be reduced to that of XY horizontal plane dynamics only. For this purpose, we assume that the vehicle is equipped with a pressure depth sensor of sufficient accuracy such that slant-range pseudo-ranges can be projected onto the horizontal plane. This also requires that the ship's trajectory never pass directly over top the vehicle, which would otherwise introduce a singularity into the horizontal range projection.

4) Fourth, that the vehicle is capable of measuring its own dead-reckoned XY odometry in-between OWTT pings, and associated measurement covariance. For example, this could be obtained from bottom-lock Doppler velocity data, an onboard INS system, or a vehicle dynamic model.

5) Finally, in the current formulation, we assume that all OWTT pseudo-range measurements occur between surface ship and vehicle only. At present, we do not consider inter-vehicle OWTT ranging. Hence, it is sufficient that each vehicle only track ship trajectory for the purposes of self-localization.

\section{B. State Description}

We denote topside ship trajectory as $\mathbf{x}_{s}(t)$, where $\mathbf{x}_{s}(t)=$ $\left[x_{s}(t), y_{s}(t)\right]^{\top}$ represents $\mathrm{XY}$ ship transducer position in a locally-defined Cartesian coordinate frame (e.g., Universal Transverse Mercator (UTM) coordinates). Similarly, we denote bottomside vehicle trajectory as $\mathbf{x}_{v}(t)=$ $\left[x_{v}(t), y_{v}(t)\right]^{\top}$. In the aforementioned scenario, the ship initiates a OWTT broadcast at time $t_{s}$ while the vehicle receives that same broadcast at a corresponding latter time $t_{v}>t_{s}$. For a given OWTT range measurement, $z_{r_{i}}$, indexed by subscript $i$, we note that this measurement actually corresponds to a ship/vehicle sample pair, $\mathbf{x}_{s_{i}}$ and $\mathbf{x}_{v_{i}}$, each sampled at distinct times $\mathbf{x}_{s_{i}}=\mathbf{x}_{s}\left(t_{s_{i}}\right)$ and $\mathbf{x}_{v_{i}}=\mathbf{x}_{v}\left(t_{v_{i}}\right)$, respectively. For notational convenience, we drop the explicit dependence on time and instead implicitly embed sample time within the $\mathbf{x}_{s_{i}}$ and $\mathbf{x}_{v_{i}}$ sample index notation. Fig. 2

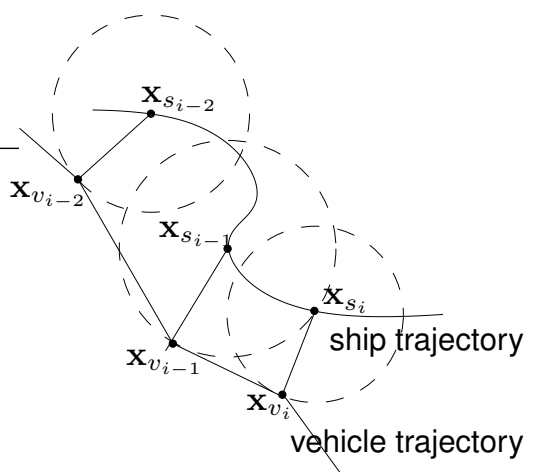

(a)

\begin{tabular}{|l|l|}
\hline Measurement Origin & Observation Model \\
\hline \hline OWTT Pseudo-Range & $\mathbf{z}_{r_{i}}=\left\|\mathbf{x}_{v_{i}}-\mathbf{x}_{s_{i}}\right\|+w_{r_{i}}$ \\
Ship GPS Position & $\mathbf{z}_{g_{i}}=\mathbf{x}_{s_{i}}+\mathbf{w}_{g_{i}}$ \\
Vehicle Odometry & $\mathbf{z}_{o_{i}}=\left(\mathbf{x}_{v_{i}}-\mathbf{x}_{v_{i-1}}\right)+\mathbf{w}_{o_{i}}$ \\
\hline
\end{tabular}

(b)

Fig. 2. MLE formulation. (a) A depiction of the 2D OWTT range geometry where $\mathbf{x}_{s_{i}}$ and $\mathbf{x}_{v_{i}}$ denote corresponding samples from the ship and vehicle trajectories, respectively. (b) A table of the available measurements and their relation to state entries. The $w_{r_{i}}, \mathbf{w}_{g_{i}}$, and $\mathbf{w}_{o_{i}}$ terms each represent additive measurement noise.

depicts a sequence of OWTT pseudo-range measurements occurring between ship and vehicle.

Next, we write our measurement observation models in terms of this sample index state description.

1) OWTT Pseudo-Range Observation Model: As stated earlier, we use measured vehicle depth to horizontally project our raw TOF pseudo-range measurements to the XY horizontal plane. Using this simplification, we write the horizontal range measurement as:

$$
z_{r_{i}}=\left\|\mathbf{x}_{v_{i}}-\mathbf{x}_{s_{i}}\right\|+w_{r_{i}}
$$

where $w_{r_{i}}$ is an additive noise term that accounts for measurement error. For the current exposition we model $w_{r_{i}}$ as being normally distributed with $w_{r_{i}} \sim \mathcal{N}\left(0, \Sigma_{r_{i}}\right)$ with $\mathrm{E}\left[w_{r_{i}} w_{r_{j}}\right]=0$ for all $i \neq j$.

2) GPS-derived Ship Position Observation Model: We first transform raw GPS measured latitude and longitude to the locally referenced $\mathrm{XY}$ coordinate system and then linearly interpolate to sample time $t_{s_{i}}$. Based upon this we write our ship GPS observation model as:

$$
\mathbf{z}_{g_{i}}=\mathbf{x}_{s_{i}}+\mathbf{w}_{g_{i}}
$$

where $\mathbf{w}_{g_{i}}$ is an additive noise term that accounts for measurement error. We model this as being normally distributed with $\mathbf{w}_{g_{i}} \sim \mathcal{N}\left(0, \Sigma_{g_{i}}\right)$ and $\mathrm{E}\left[\mathbf{w}_{g_{i}} \mathbf{w}_{g_{j}}^{\top}\right]=0$ for all $i \neq j$.

3) Vehicle-derived Odometry Observation Model:

Vehicle-derived odometry measurements represent the inter-ping vector displacement between OWTT fixes and are a necessary constraint in order to establish vehicle observability when dealing with single-transponder ranges [18], [20]. We write our odometry observation model as:

$$
\mathbf{z}_{o_{i}}=\mathbf{x}_{v_{i}}-\mathbf{x}_{v_{i-1}}+\mathbf{w}_{o_{i}}
$$


where $\mathbf{w}_{o_{i}}$ is an additive noise term that accounts for measurement error. We model the error as being normally distributed with $\mathbf{w}_{o_{i}} \sim \mathcal{N}\left(0, \Sigma_{o_{i}}\right)$ and $\mathrm{E}\left[\mathbf{w}_{o_{i}} \mathbf{w}_{o_{j}}^{\top}\right]=0$ for all $i \neq j$.

\section{MLE Optimization}

We pose sensor fusion as a maximum likelihood estimate (MLE) optimization problem. In this context, we treat ship/vehicle sample pairs as unknown parameters that we wish to estimate. To begin, we consider the case of $n$ range measurements and define the following:

$\mathbf{X}_{v}=\left\{\mathbf{x}_{v_{i}}\right\}_{0}^{n-1}$ is the set of vehicle trajectory samples,

$\mathbf{X}_{s}=\left\{\mathbf{x}_{s_{i}}\right\}_{0}^{n-1}$ is the set of ship trajectory samples,

$\mathbf{Z}_{r}=\left\{z_{r_{i}}\right\}_{0}^{n-1}$ is the set of pseudo-range measurements,

$\mathbf{Z}_{g}=\left\{\mathbf{z}_{g_{i}}\right\}_{0}^{n-1}$ is the set of ship GPS measurements,

$\mathbf{Z}_{o}=\left\{\mathbf{z}_{o_{i}}\right\}_{1}^{n-1}$ is the set of odometry measurements,

with $\mathbf{X}=\left\{\mathbf{X}_{v}, \mathbf{X}_{s}\right\}$ and $\mathbf{Z}=\left\{\mathbf{Z}_{r}, \mathbf{Z}_{g}, \mathbf{Z}_{o}\right\}$.

Denoting the measurement likelihood as $L(\mathbf{X})$ we have

$$
\begin{aligned}
L(\mathbf{X}) & =p(\mathbf{Z} \mid \mathbf{X}) \\
& =p\left(\mathbf{Z}_{r} \mid \mathbf{X}_{v}, \mathbf{X}_{s}\right) p\left(\mathbf{Z}_{g} \mid \mathbf{X}_{s}\right) p\left(\mathbf{Z}_{o} \mid \mathbf{X}_{v}\right),
\end{aligned}
$$

where the mutual independence of measurements on parameters is explicit. To optimize, we wish to find

$$
\hat{\mathbf{X}}=\arg \max _{\mathbf{X}} p(\mathbf{Z} \mid \mathbf{X})
$$

which is equivalent to solving

$$
\hat{\mathbf{X}}=\arg \min _{\mathbf{X}}-\ln p(\mathbf{Z} \mid \mathbf{X}) .
$$

Under the assumed observation models and noise statistics, we can write our objective function, $C(\mathbf{X})$, as

$$
\begin{aligned}
& C(\mathbf{X})=-\ln p(\mathbf{Z} \mid \mathbf{X}) \\
= & \frac{1}{2} \sum_{i=0}^{n-1}\left(z_{r_{i}}-\left\|\mathbf{x}_{v_{i}}-\mathbf{x}_{s_{i}}\right\|\right)^{\top} \Sigma_{r_{i}}^{-1}\left(z_{r_{i}}-\left\|\mathbf{x}_{v_{i}}-\mathbf{x}_{s_{i}}\right\|\right) \\
+ & \frac{1}{2} \sum_{i=0}^{n-1}\left(\mathbf{z}_{g_{i}}-\mathbf{x}_{s_{i}}\right)^{\top} \Sigma_{g_{i}}^{-1}\left(\mathbf{z}_{g_{i}}-\mathbf{x}_{s_{i}}\right) \\
+ & \frac{1}{2} \sum_{i=1}^{n-1}\left(\mathbf{z}_{o_{i}}-\left(\mathbf{x}_{v_{i}}-\mathbf{x}_{v_{i-1}}\right)\right)^{\top} \Sigma_{o_{i}}^{-1}\left(\mathbf{z}_{o_{i}}-\left(\mathbf{x}_{v_{i}}-\mathbf{x}_{v_{i-1}}\right)\right) .
\end{aligned}
$$

To optimize, we recognize that (4) can be more compactly written as

$$
C(\mathbf{X})=\left(\mathbf{Z}-\mathbf{h}_{\mathbf{Z}}(\mathbf{X})\right)^{\top} \Sigma_{\mathbf{Z}}^{-1}\left(\mathbf{Z}-\mathbf{h}_{\mathbf{Z}}(\mathbf{X})\right)
$$

where $\mathbf{h}_{\mathbf{Z}}(\mathbf{X})$ is the stacked vector of observations and $\Sigma_{\mathbf{Z}}$ is the block-diagonal measurement covariance. In this form, it is clear that our objective function results in a nonlinear weighted least-squares optimization problem. To solve, we employ the Levenberg-Marquardt algorithm [23] starting with an initial guess of dead-reckoned (DR) vehicle position and GPS-measured ship position.

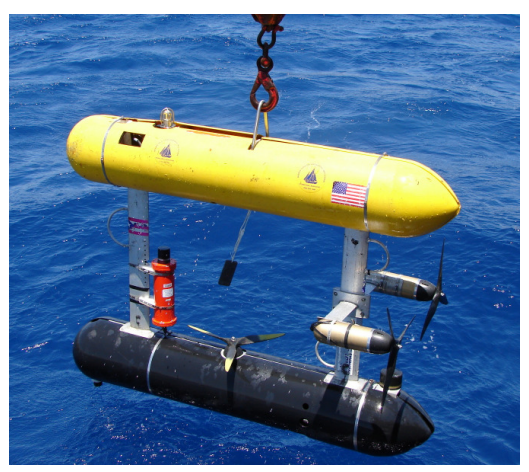

Fig. 3. The SeaBED AUV used during field experiments [25]. The orange Tracklink beacon mounted on the front strut provided an independent measurement of ship-to-vehicle range.

To obtain a first-order estimate of parameter covariance, $\Sigma_{\mathbf{X}}$, we compute

$$
\Sigma_{\mathbf{X}}=\left(\mathrm{J}^{\top} \Sigma_{\mathbf{Z}}^{-1} \mathrm{~J}\right)^{-1}
$$

where $\mathrm{J}$ is the Jacobian evaluated at the optima [24]:

$$
\mathrm{J}=\left.\frac{\mathrm{d} \mathbf{h}_{\mathbf{Z}}(\mathbf{X})}{\mathrm{d} \mathbf{X}}\right|_{\hat{\mathbf{X}}} .
$$

\section{RESUlTS}

In this section we report results for two sets of field experiments employing OWTT navigation with MLE sensor fusion. Both experiments were for a two-node network consisting of the tending surface ship and a single AUV. In each case, the SeaBED AUV platform (Fig. 3) was used.

\section{A. Experimental Setup}

The SeaBED AUV is instrumented with a typical suite of oceanographic navigation sensors including pressure sensor depth, $1200 \mathrm{kHz}$ DVL body-frame velocities, an IXSEA North-seeking 3-axis fiber optic gyro for attitude, and a PPS-capable WHOI Micro-modem [25]. In addition, we integrated our PPSBOARD and a Garmin GPS-16HVS GPS receiver into the vehicle system so that we could conduct OWTT experiments. The Garmin GPS unit outputs a 1 PPS reference signal accurate to within $1 \mu$ s of UTC when it has GPS lock. We use this PPS reference for pre-dive time synchronization of the free running TXCO onboard the AUV while at the surface.

The surface ship is equipped with a PPS-capable Micromodem, a GPS receiver used for measuring ship transducer position, and a Meinberg GPS-based NTP time server as a stable clock reference. The Meinberg unit outputs a 1 PPS signal accurate to within $100 \mathrm{~ns}$ and is drift free.

\section{B. Experimental Results}

1) Experiment 1 - GPS Validation: During December of 2005, we operated the SeaBED AUV offshore the coast of Woods Hole, MA using the R/V Tioga. For this set of experiments we deployed the AUV in approximately $15 \mathrm{~m}$ of water and programed the vehicle to swim two $100 \mathrm{~m}$ concentric boxes at a forward speed of $0.4 \mathrm{~m} / \mathrm{s}$. In this environment, 


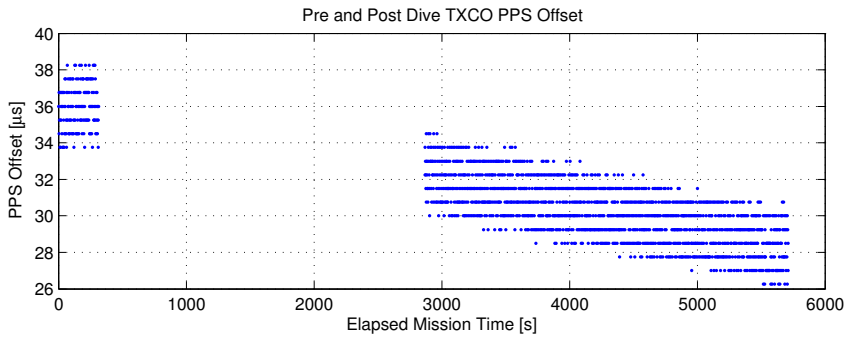

Fig. 4. Pre and post-dive measured TXCO PPS offset with respect to GPSderived absolute PPS timebase. During the course of the dive, the TXCO drifted by approximately $4 \mu \mathrm{s}$ from an initial offset of $36 \mu$ s to $32 \mu \mathrm{s}$.

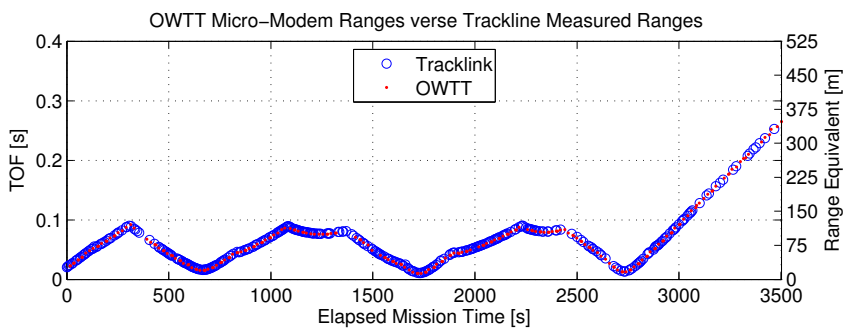

(a)

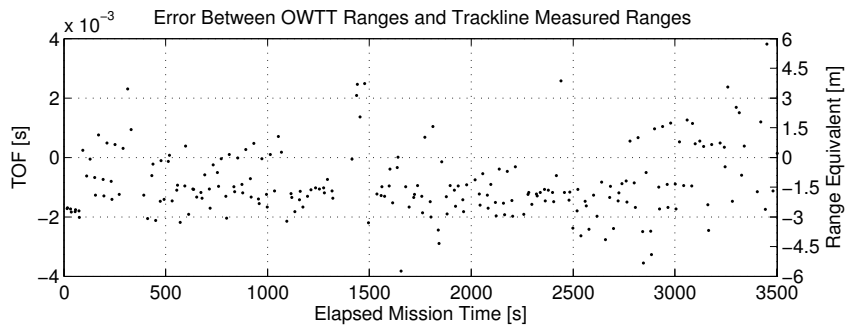

(b)

Fig. 5. A comparison of modem-derived OWTT ranges versus USBLderived TWTT ranges measured between ship and AUV. (a) A plot of raw ranges as measured by the the Tracklink USBL system and modem-derived OWTTs. (b) A comparison of the TOF discrepancy obtained by linearly interpolating the USBL range data to the OWTT timebase. For reference, the $y$-axis scale on the right shows the equivalent range error assuming a sound speed of $1500 \mathrm{~m} / \mathrm{s}$.

the AUV had Doppler bottom-lock from the surface with a total mission time of approximately 1.5 hours, and a PPS clock-drift of less than $4 \mu$ s onboard the AUV (Fig. 4). The ship remained on anchor while broadcasting OWTT pings to the AUV at a schedule of 0,14 , and 36 seconds past the top of the minute. Fig. 5 displays the raw OWTT data recorded between the AUV and ship; for comparison, independently measured Tracklink USBL TWTT ranges are shown.

A random-walk model including the effect of first-order heading uncertainty was used to calculate inter-ping DVL $\mathrm{XY}$ odometry covariance using settings of $3 \mathrm{~cm} / \mathrm{s}$ standard deviation for the body-frame surge/sway velocities (assumed isotropic) and $0.1^{\circ}$ standard deviation for the heading; these numbers were obtained from the respective manufacturer's manuals as being typical precisions. We set the OWTT ranges at $18.75 \mathrm{~cm}$ standard deviation, based upon the Micro-modem's $125 \mu \mathrm{s}$ TOA detection resolution. For ship GPS position error, we used the horizontal error estimated

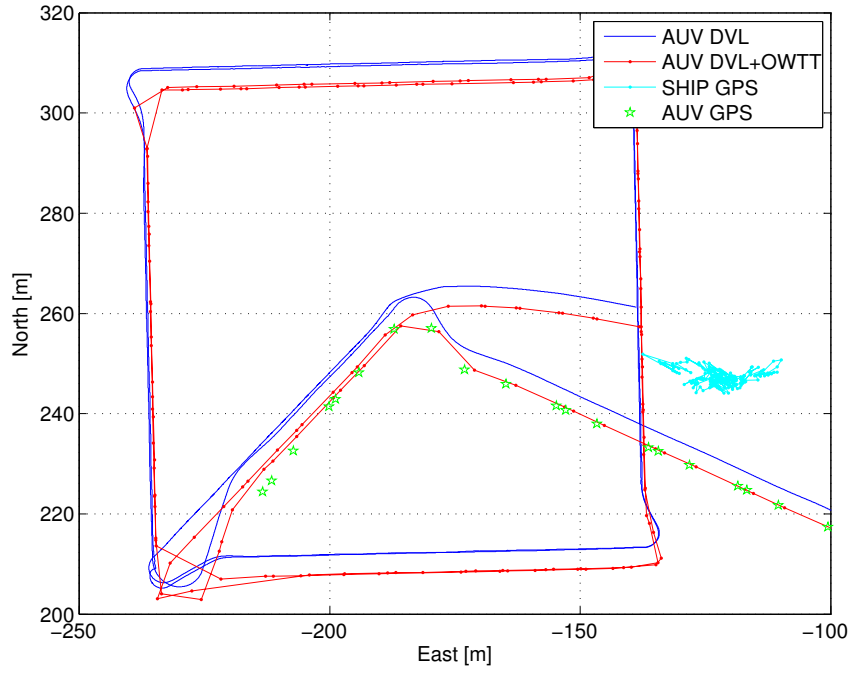

(a)

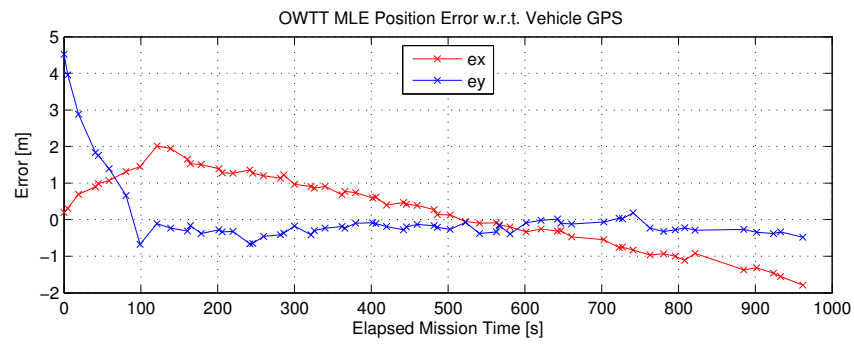

(b)

Fig. 6. MLE sensor fusion results for a two node network consisting of a bottom-lock Doppler-aided AUV and a GPS-equipped surface ship. Each node is equipped with a WHOI Micro-Modem and onboard stable PPS source for synchronous clock communication/navigation. (a) The AUV swam two co-located box trajectories ( $100 \mathrm{~m}$ per side), each at a differen depth set point while the ship remained anchored. Shown in blue is the raw DVL-derived AUV trajectory; in cyan is the GPS-derived ship position; in red is the globally referenced MLE AUV trajectory; and in green is the end-of-dive GPS-measured AUV position, which serves as an independent ground-truth. (b) Shown here is the error between end-of-dive OWTTderived and GPS-measured AUV position.

by the receiver and assumed it to be isotropic.

Fig. 6 displays results from the MLE fusion of the OWTT pseudo-ranges and bottom-lock DVL odometry. The raw DVL trajectory, shown in blue, was obtained by forward Euler integration, using the GPS drop-position of the AUV as the origin. Shown in red is the globally referenced MLE derived trajectory; this result was post-processed offline. Shown in green is the independently measured post-dive GPS position of the AUV, which cross-validates the OWTT result. Fig. 6(b) provides a plot of GPS referenced error versus time, showing good agreement between OWTT derived position and onboard GPS.

2) Experiment 2 - LBL Validation: In July of 2006, we operated aboard the Greek vessel R/V Aegeo as part of a joint WHOI / Hellenic Centre for Marine Research (HCMR) research cruise in the Mediterranean. This time we performed a longer series of OWTT experiments with the SeaBED AUV, deploying it for a mission just under 2 hours 
in duration. The water depth was approximately $50 \mathrm{~m}$ deep and the programmed survey trajectory consisted of two gridpatterns: one oriented East-to-West and the other North-toSouth. The survey bounding box was approximately $200 \mathrm{~m}$ on a side. In addition, we deployed a two-transponder LBL net for independent position validation.

Fig. 7 shows results comparing raw DVL, MLE-fused OWTT data, and cross-validated $12 \mathrm{kHz}$ LBL vehicle position. For this experiment, the surface ship free-drifted about the survey site, occasionally motoring to get back on station. Its trajectory is depicted by the cyan curve in Fig. 7(a). Fig. 7(b) shows a close-up view of the re-navigated survey. The coarse agreement between OWTT MLE and LBL is visually evident, in contrast with the raw Doppler track whose error increases with time. Figures $7(\mathrm{c}) / 7$ (d) and 7(e)/7(f) show that the raw DVL estimate exhibits a timedependent bias while the OWTT re-navigated trajectory does not.

\section{CONCLUSIONS}

This paper reported recent results in the development of a synchronous-clock acoustic communication/navigation system for underwater vehicles. The long-term goal of this work is to enable the task of navigating a fleet of AUVs over order $100 \mathrm{~km}$ length scales, with bounded-error commensurate with standard $12 \mathrm{kHz}$ LBL navigation. Toward that end, we have reported the development of a low power, stable clock system suitable for integration on AUVs. We have also established a preliminary maximum-likelihood fusion framework for combining OWTT pseudo-range measurements with vehicle-odometry for bounded-error navigation. Results from two field experiments validating the OWTT MLE framework were reported. Future research in this area will address the modeling of PPS clock drift, the use of water-lock DVL velocity odometry, and a decentralized estimation framework to support multi-vehicle navigation using inter-vehicle ranging.

\section{ACKNOWLEDGMents}

This work was supported by the National Science Foundation under Award Numbers ATM-0427220 and ATM0428122, and in part by the National Oceanic and Atmospheric Administration Office of Ocean Exploration under Grant Number NA06OAR4600092.

\section{REFERENCES}

[1] L. Whitcomb, D. Yoerger, and H. Singh, "Towards precision robotic maneuvering, survey and manipulation in unstructured undersea environments," in Proc. Intl. Symp. Robotics Research, Springer Verlag, London, 1998, pp. 45-54.

[2] M. Hunt, W. Marquet, D. Moller, K. Peal, W. Smith, and R. Spindel, "An acoustic navigation system," Woods Hole Oceanographic Institution, Tech. Rep. WHOI-74-6, Dec. 1974.

[3] P. Milne, Underwater acoustic positioning systems. Houston: Gulf Publishing Company, 1983.

[4] J. Bellingham, M. Deffenbaugh, J. Leonard, and J. Catipovic, "Arctic under-ice survey operations," Unmanned Systems, vol. 12, pp. 24-29, 1994.
[5] H. Singh, J. Catipovic, R. Eastwood, L. Freitag, H. Henriksen, F. Hover, D. Yoerger, J. Bellingham, and B. Moran, "An integrated spproach to multiple AUV communications, navigation and docking," in Proc. IEEE/MTS OCEANS Conf. Exhib., vol. 1, Fort Lauderdale, Sept. 1996, pp. 59-64.

[6] S. Smith and D. Kronen, "Experimental results of an inexpensive short baseline acoustic positioning system for AUV navigation," in Proc. IEEE/MTS OCEANS Conf. Exhib., vol. 1, Halifax, NS, 1997, pp. 714720.

[7] M. Larsen, "High performance Doppler-inertial navigationexperimental results," in Proc. IEEE/MTS OCEANS Conf. Exhib., vol. 2, Providence, RI, 2000, pp. 1449-1456.

[8] T. Gaiffe, "U-Phins: a FOG-based inertial navigation system developed specifically for AUV navigation and control," in Intl. Conf. Underwater Intervention, New Orleans, LA, Feb. 2002.

[9] J. Kinsey, R. Eustice, and L. Whitcomb, "Underwater vehicle navigation: recent advances and new challenges," in IFAC Conf. on Manoeuvring and Control of Marine Craft, Lisbon, Portugal, Sept. 2006, Accepted, To Appear.

[10] L. Freitag, M. Grund, S. Singh, J. Partan, P. Koski, and K. Ball, "The WHOI micro-modem: an acoustic communications and navigation system for multiple platforms," in Proc. IEEE/MTS OCEANS Conf. Exhib., Washington, D.C., Sept. 2005.

[11] L. Freitag, M. Grund, J. Partan, K. Ball, S. Singh, and P. Koski, "Multi-band acoustic modem for the communications and navigation aid AUV," in Proc. IEEE/MTS OCEANS Conf. Exhib., Washington, D.C., Sept. 2005.

[12] J. Curcio, J. Leonard, J. Vaganay, A. Patrikalakis, A. Bahr, D. Battle, H. Schmidt, and M. Grund, "Experiments in moving baseline navigation using autonomous surface craft," in Proc. IEEE/MTS OCEANS Conf. Exhib., Washington, D.C., Sept. 2005.

[13] H. Singh, J. Bellingham, F. Hover, S. Lerner, B. Moran, K. von der Heydt, and D. Yoerger, "Docking for an autonomous ocean sampling network," IEEE J. Oceanic Eng., vol. 26, no. 4, pp. 498-514, Oct. 2001.

[14] A. Scherbatyuk, "The AUV positioning using ranges from one transponder LBL," in Proc. IEEE/MTS OCEANS Conf. Exhib., vol. 3, San Diego, CA, 1995, pp. 1620-1623.

[15] M. Larsen, "Synthetic long baseline navigation of underwater vehicles," in Proc. IEEE/MTS OCEANS Conf. Exhib., vol. 3, Sept. 2000, pp. 2043-2050.

[16] J. Vaganay, P. Baccou, and B. Jouvencel, "Homing by acoustic ranging to a single beacon," in Proc. IEEE/MTS OCEANS Conf. Exhib., vol. 2, Sept. 2000, pp. 1457-1462.

[17] P. Baccou and B. Jouvencel, "Homing and navigation using one transponder for AUV, postprocessing comparisons results with long base-line navigation," in Proc. IEEE Intl. Conf. Robot. Auto., vol. 4, 2002, pp. 4004-4009.

[18] A. Gadre and D. Stilwell, "Toward underwater navigation based on range measurements from a single location," in Proc. IEEE Intl. Conf. Robot. Auto., vol. 5, 2004, pp. 4472-4477.

[19] — - "A complete solution to underwater navigation in the presence of unknown currents based on range measurements from a single location," in Proc. IEEE/RSJ Intl. Conf. Intell. Robots Systems, 2005, pp. $1420-1425$.

[20] A. Ross and J. Jouffroy, "Remarks on the observability of single beacon underwater navigation," in Proc. Intl. Symp. Unmanned Unteth. Subm. Tech., Aug. 2005.

[21] S. Singh, M. Grund, B. Bingham, R. Eustice, H. Singh, and L. Freitag, "Underwater acoustic navigation with the WHOI micro-modem," in Proc. IEEE/MTS OCEANS Conf. Exhib., Boston, MA, Sept. 2006, Accepted, To Appear.

[22] D. Mills, "Network time protocol version 4 reference and implementation guide," University of Delaware, Tech. Rep. 06-06-1, June 2006.

[23] W. Press, S. Teukolsky, W. Vetterling, and B. Flannery, Numerical recipes in $C$ : the art of scientific computing, 2 nd ed. Cambridge University Press, 1992.

[24] R. Hartley and A. Zisserman, Multiple view geometry in computer vision. Cambridge University Press, 2000.

[25] H. Singh, A. Can, R. Eustice, S. Lerner, N. McPhee, O. Pizarro, and C. Roman, "SeaBED AUV offers new platform for high-resolution imaging," EOS, Trans. Amer. Geophysical Union, vol. 85, no. 31, pp. 289,294-295, Aug. 2004. 


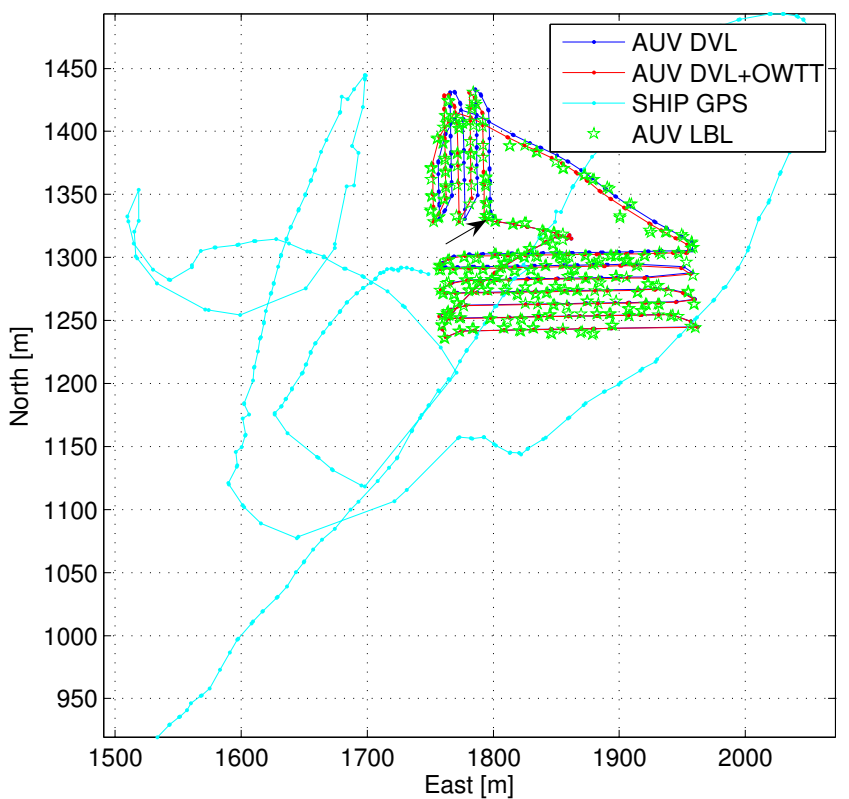

(a)

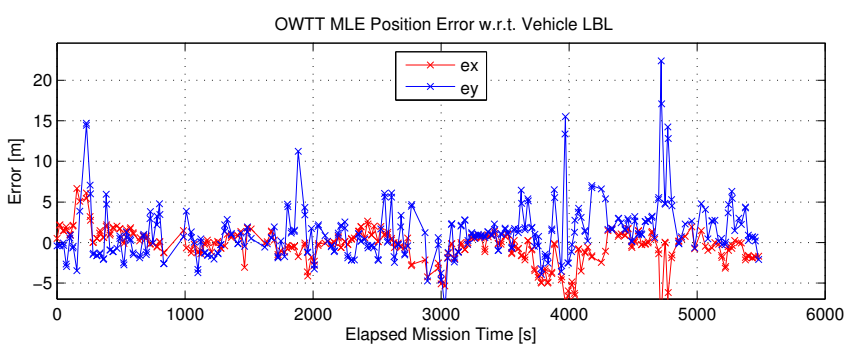

(c)
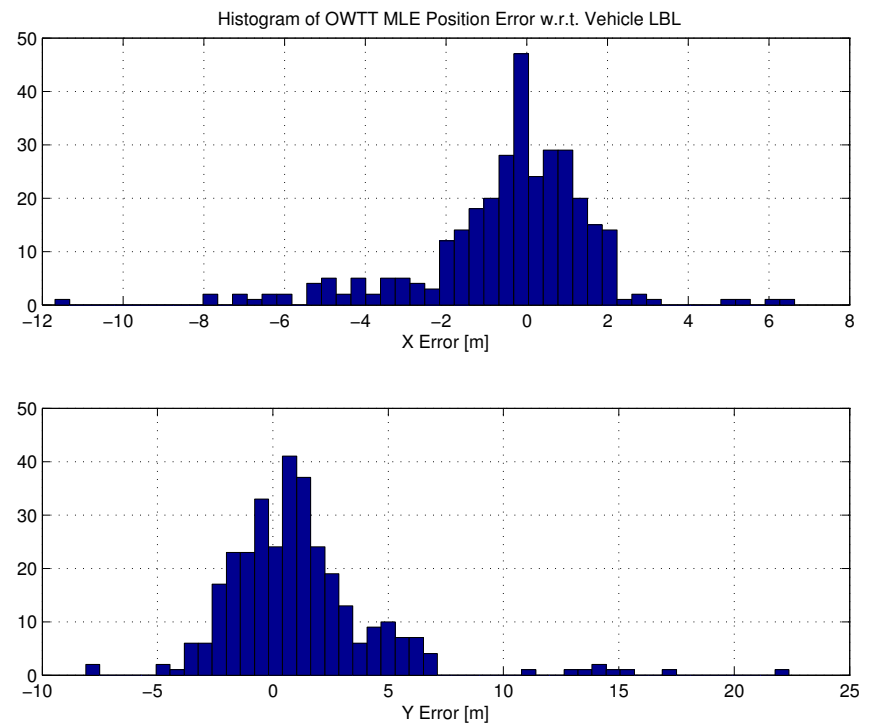

(e)

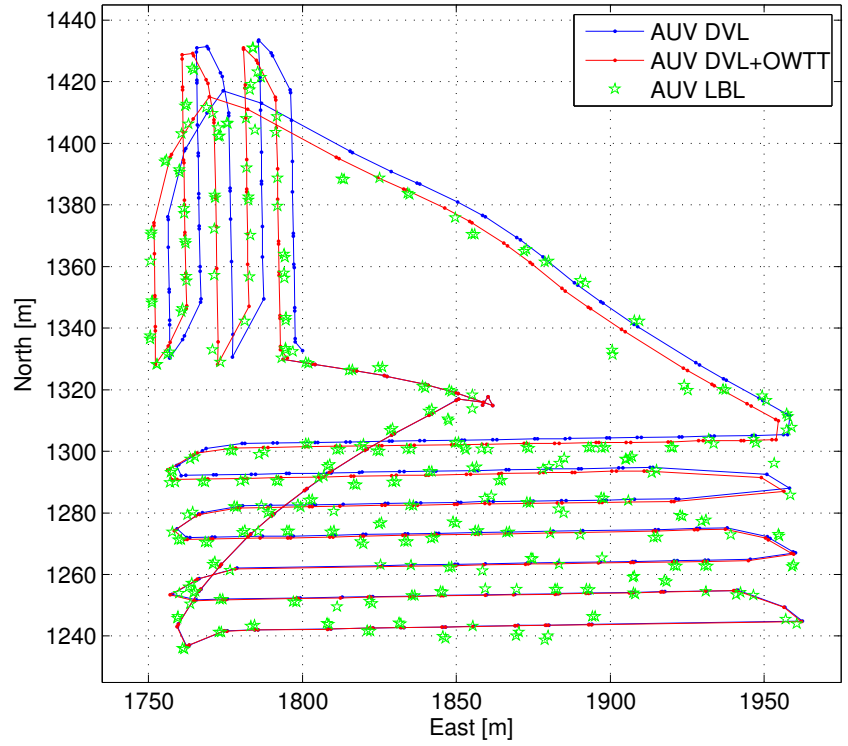

(b)

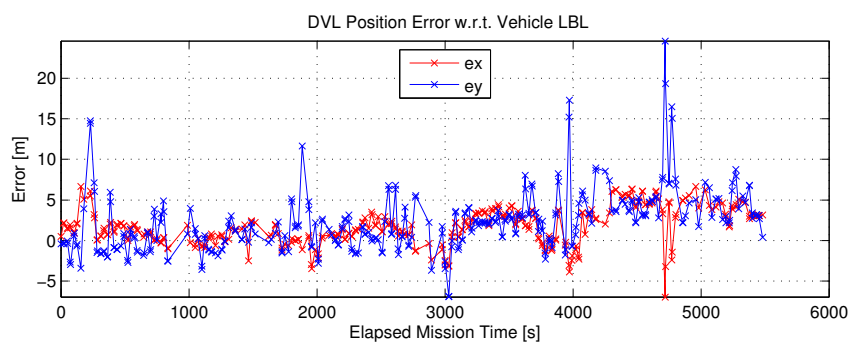

(d)
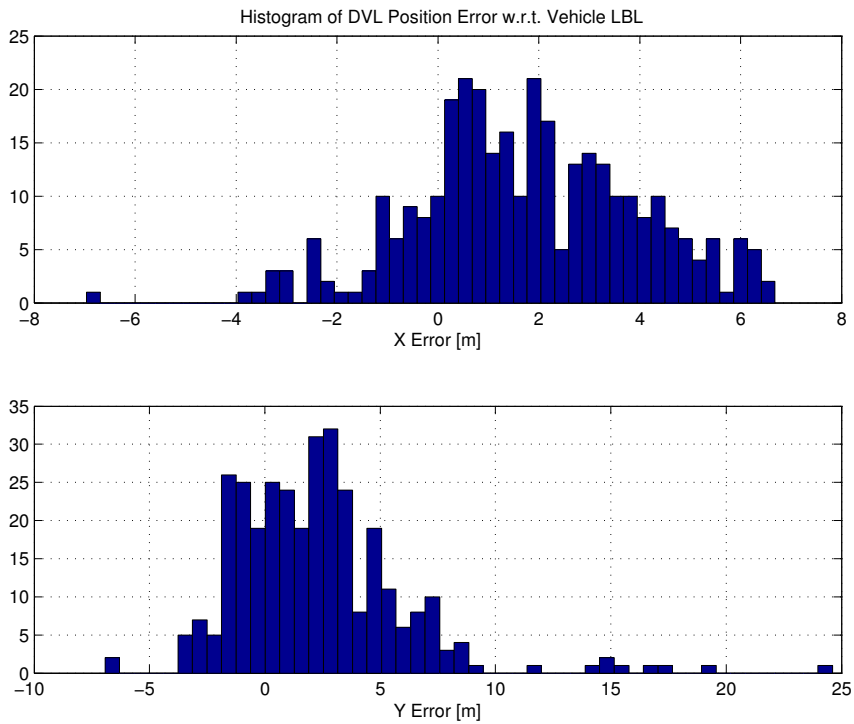

(f)

Fig. 7. MLE sensor fusion results for a two-node configuration consisting of a GPS-equipped surface ship and bottom-lock Doppler-aided AUV. The survey took place in approximately $50 \mathrm{~m}$ of water depth. (a) The programed vehicle trajectory consisted of two grid-survey patterns: one oriented East-to-West and the other North-to-South. Shown in blue is the raw DVL-derived DR AUV trajectory; in cyan is the GPS-derived ship position; in red is the globally referenced MLE AUV trajectory; and in green is $12 \mathrm{kHz}$ LBL-derived AUV position, which serves as an independent ground-truth. The mission starts and ends at approximately $(1794,1330)$, as indicated by the arrow. (b) A close up view of the vehicle trajectory. The discrepancy between raw DVL position and LBL increases with time. In contrast, the OWTT-aided trajectory agrees well with the independently measured LBL position. (c) and (d) These two plots show OWTT and raw DVL position error versus elapsed mission time; error is defined with respect to LBL. Again, note the zero-mean nature of OWTT-derived position versus the time dependent DVL error. X and Y error histograms are shown in (e) and (f). 